\title{
A dual frequency, ultrasonic, microengineered particle manipulator
}

\author{
N. Harris ${ }^{\mathrm{a}, *}$, M. Hill ${ }^{\mathrm{b}}$, Y. Shen ${ }^{\mathrm{b}}$, R.J. Townsend ${ }^{\mathrm{b}}, \mathrm{S}$. Beeby ${ }^{\mathrm{a}}$, N. White ${ }^{\text {a }}$ \\ ${ }^{a}$ ECS, Southampton University, Highfield, Mountbatten 3007, Southampton SO17 1BJ, UK \\ b SES, Southampton University, Southampton SO17 1BJ, UK
}

\begin{abstract}
Ultrasonic standing waves can be used to generate forces on particles within a fluid. Recent work has concentrated on developing devices that manipulate the particles so that they are concentrated near the centre of the cavity. It is also possible to design a device that concentrates the particles at the wall of a cavity. This paper describes a device that has the capability of operating in several modes to allow concentration of particles at either the cavity wall or the centre of the cavity, depending on the driving frequency. (C) 2004 Elsevier B.V. All rights reserved.
\end{abstract}

Keywords: Ultrasonics; Microfluidics; Particle manipulation

\section{Introduction}

High frequency acoustic standing waves can be used to separate materials with different acoustic characteristics. The technique can be used to agglomerate particles, to hold particles within a flow, or to manipulate particles with the flow [1].

This paper describes the modelling of such a device and shows how the model has been used to design a chamber that works efficiently at a number of different modal frequencies allowing particles to be concentrated at different points across the cavity depth, depending on the driving frequency.

\section{Modelling and construction of the device}

The system can be modelled as a multilayered resonant structure as described by Hill et al. [2,3]. The modelling approach has been used successfully in the design of a fluid clarifier (a device which allows a sample of particlefree fluid to be extracted from a flow), shown schematically in Fig. 1. A PZT transducer is attached to a silicon carrier layer in which inlet and outlet ports are etched. The silicon is anodically bonded to a Pyrex wafer into which a fluid filled cavity is etched. This clarifier operates

\footnotetext{
${ }^{*}$ Corresponding author. Tel.: +44-2380-593274; fax: +44-2380592901.

E-mail address: nrh@ecs.soton.ac.uk (N. Harris).
}

in a half-wave mode, in which the cavity depth is half the acoustic wavelength at resonance, creating a pressure node at which particles gather in the centre of the cavity. The dimensions of the structure were chosen so that the device operated efficiently at a frequency of about 2.4 $\mathrm{MHz}$, with a cavity depth of the order of $240 \mu \mathrm{m}$.

The device described in this paper extends this concept, as it allows particles to be positioned at different planes within the cavity, depending on the driving frequency. The device is fabricated in silicon and pyrex, and as such is able to exploit standard silicon processes. In addition, the pyrex allows the cavity itself to be viewed. Driving is achieved via a bonded PZT plate.

The motivation for the work came from two sources:

- experimental observations from Hawkes et al. [4] that for certain combinations of layer geometries, particles can be forced up against the reflector layer, implying a pressure minimum within the fluid lies at the solid boundary - a quarter-wave mode,

- a theoretical prediction [5] that for particular combinations of chamber and reflector layer depths, both half-wave and quarter-wave modes can exist.

The feature of the quarter-wave device mode that is of significant interest is the ability to drive particles to the pyrex boundary. This mode has application in biosensors, where it may be possible to improve the sensitivity of such sensors by concentrating the active element on the sense element. The half wave cavity mode 


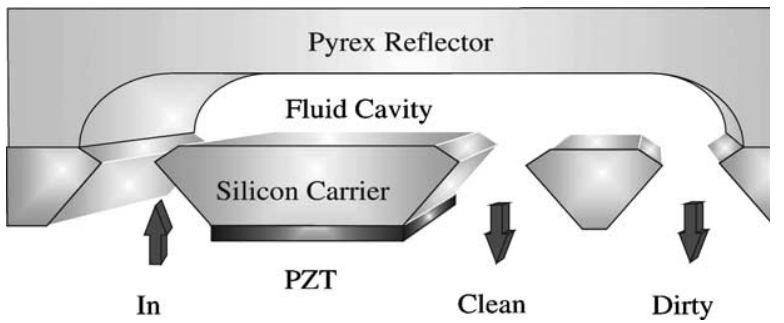

Fig. 1. Cross section of fluid clarifier.

has application in particle separators, and is the mode that can be generated most efficiently.

Theoretical predictions based on the cavity and reflector alone predict that an efficient quarter-wave mode will exist in the cavity if a frequency exists for which the cavity depth is a quarter wavelength and the reflector depth is a half wavelength. This is described in Eq. (1), where $c_{\mathrm{r}}, c_{\mathrm{f}}$ are the sound velocities in the reflector and fluid layers respectively and $t_{\mathrm{r}}, t_{\mathrm{f}}$ are the thicknesses of the reflector and fluid layers. The total thickness of the combined fluid and reflector layer is specified as $1.7 \mathrm{~mm}$, as this is a stock pyrex wafer size.

$\frac{c_{\mathrm{r}}}{f}=2 t_{\mathrm{r}}, \quad \frac{c_{\mathrm{f}}}{f}=4 t_{\mathrm{f}} \quad$ and $\quad t_{\mathrm{f}}+t_{\mathrm{r}}=1.7 \times 10^{-3} \mathrm{~m}$

By substituting $c_{\mathrm{r}}=5430 \mathrm{~m} \mathrm{~s}^{-1}$ and $c_{\mathrm{f}}=1500 \mathrm{~m} \mathrm{~s}^{-1}$, eliminating $f$ and then solving for the thickness of the fluid layer, it can be shown that the cavity should have a depth of $206 \mu \mathrm{m}$.

Hence a quarter wave mode should exist for a cavity depth of about $200 \mu \mathrm{m}$ with a frequency near $1.8 \mathrm{MHz}$. This cavity/reflector combination should also produce a half-wave resonance near 3.6 MHz. Simulations were carried out over a range cavity etch depths and Fig. 2 shows the energy density within the fluid layer as a function of frequency and cavity depth.

The expected "quarter wave" mode is the very narrow peak labelled "mode 1" at about 1.8 MHz. However there are two modes (modes 3 and 4), one on each

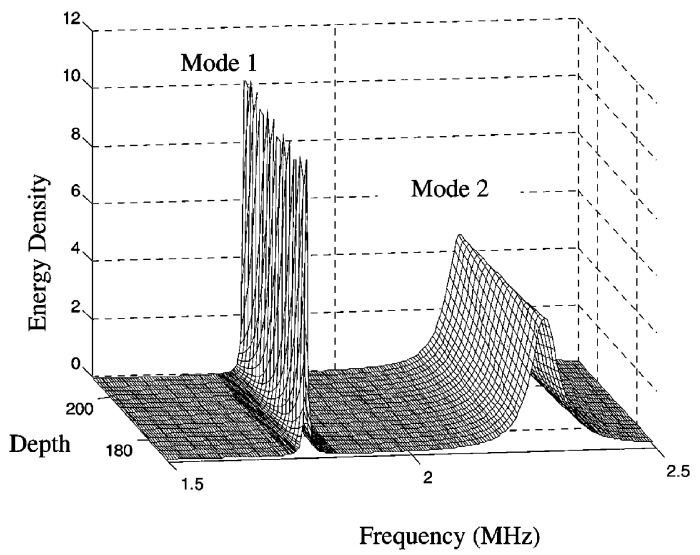

side of the expected "half wave" mode. In addition, there is a mode (mode 2) a little above $2 \mathrm{MHz}$. The nature of the modes can be explored in more depth if the pressure profiles of these modes are plotted as in Fig. 3. It can be seen that, as expected, mode 1 is approximately a quarter wavelength with a pressure maximum at the silicon boundary and a pressure minimum at or near the reflector boundary. Mode 2 is also a "quarter wave" mode, but in this case the pressure minimum is at, or near, the silicon boundary. Modes 3 and 4 are similar and both are close to a half wavelength with a pressure node near to the centre of the cavity.

As the main aim of this investigation was to demonstrate a device in which particles could be moved either to the centre or a boundary of the cavity, a cavity depth of $175 \mu \mathrm{m}$ was chosen so that both "quarter wave" modes were predicted to have their pressure minimum at, rather than simply near, the boundaries.

The fabrication technique to be used involves etching the cavity out of a Pyrex plate. The silicon wafer has a thickness of $525 \mu \mathrm{m}$.

The device was constructed in a similar manner to the half wave concentrator [6] with the exception that the cavity depth was etched to $175 \mu \mathrm{m}$ rather than the previous $250 \mu \mathrm{m}$. Inlet and outlet ports were etched in the silicon using a standard $\mathrm{KOH}$ wet etch. The bulk PZT transducer drive element was bonded onto the silicon using standard Araldite epoxy resin.

In order to allow fluid into and out of the device, a manifold was constructed from aluminium as shown in Fig. 4. The device was held on the top of the manifold with four small screw-on clamps, and the manifold/silicon seal was achieved with a custom silicon O-ring.

\section{Verification of the model}

In order to verify the model against the fabricated device, an impedance analyser was used. The instrument

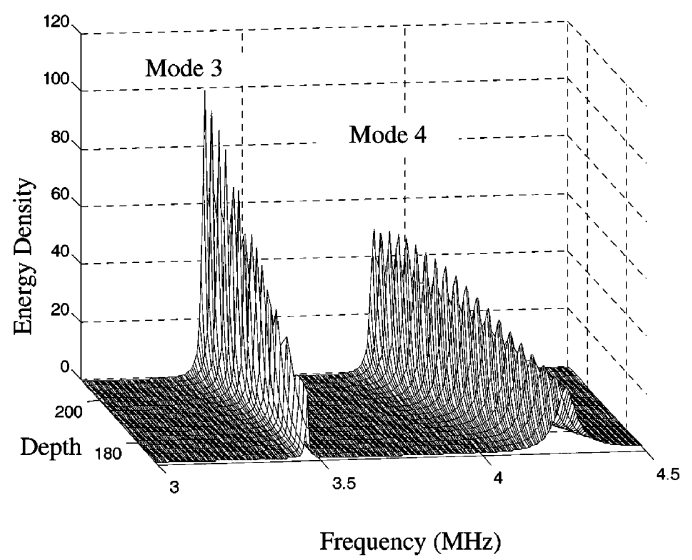

Fig. 2. Energy density $\left(\mathrm{J} \mathrm{m}^{-3}\right)$ in the cavity against depth in microns and frequency in MHz. Note different $z$-axis scales for the two frequency ranges. 

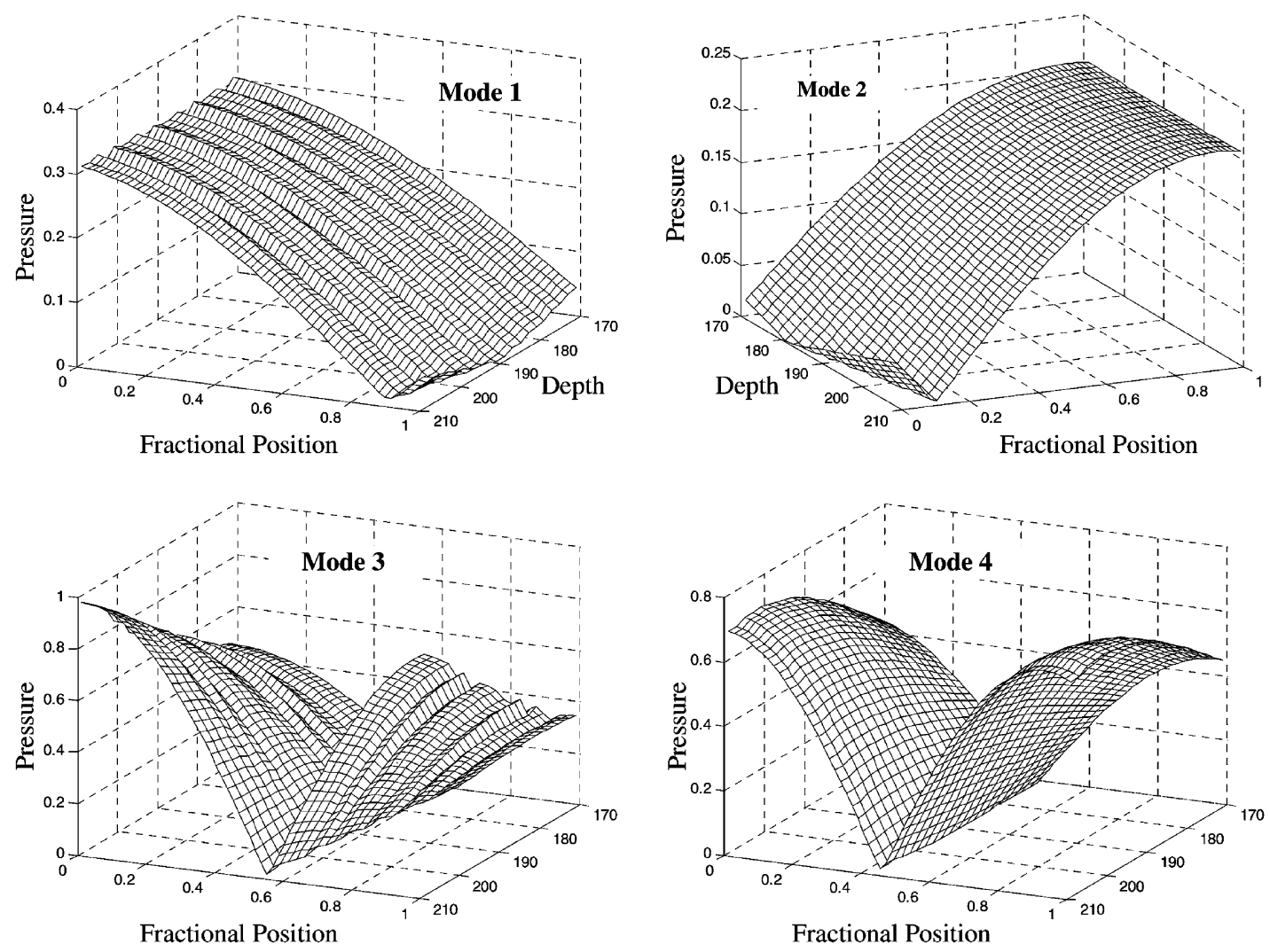

Fig. 3. Modelled pressure distributions across the cavity (fractional distance from the silicon layer) for four cavity modes shown in Fig. 2, plotted against cavity depth in microns.

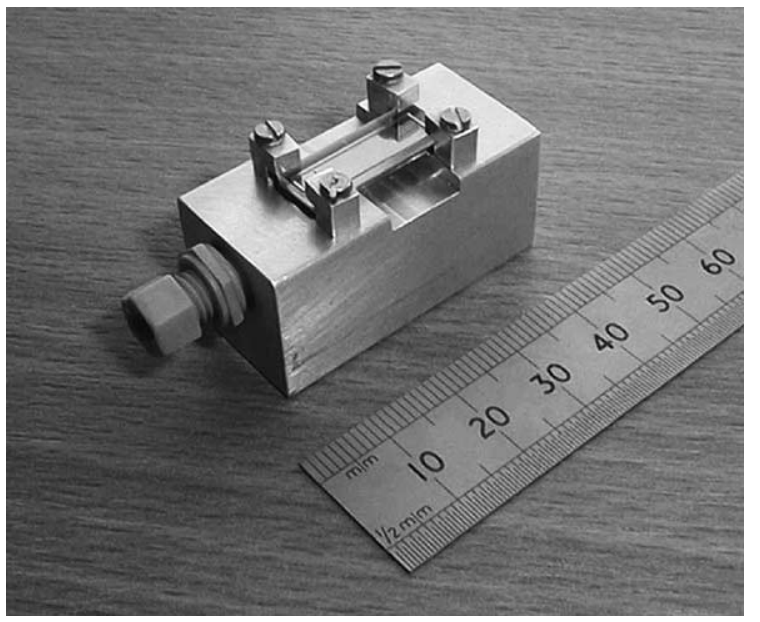

Fig. 4. The micromachined resonator on the aluminium manifold.

used was a Hewlett Packard 4192A frequency analyser, under PC control. Measurements were taken at an excitation voltage of $1 \mathrm{~V}$. The model predictions for impedance are shown in Figs. 5 and 6, together with the corresponding measured results. Fig. 5 shows the results for the chamber filled with air, and Fig. 6 shows the results for the chamber filled with fluid.
In Fig. 5, the dotted line shows the modelled response of effectively the PZT drive element coupled via a glue layer to the silicon layer. As the cavity is filled with air, this is effectively the limit of the model and so no cavity response is expected. Two resonances are expected in this configuration, one at $2.3 \mathrm{MHz}$ and a smaller one at $4.7 \mathrm{MHz}$. The solid line shows the measured impedance curve. As can be seen there is a large peak at $2.6 \mathrm{MHz}$ and a smaller peak at $4.7 \mathrm{MHz}$. There are some other artefacts present in the lower frequencies, but the origin of these is unclear at present.

Fig. 6 shows the corresponding results for the device with the cavity full of water. Although the expected cavity modes at 3.5 and $4.1 \mathrm{MHz}$ are clearly visible, those at 1.8 and $2.2 \mathrm{MHz}$ are masked by the transducer/ carrier layer resonance and other artefacts.

However, in practical terms, the modes are most easily identified by measuring the voltage drop across the transducer, and so a voltage trace was also taken, using a HP spectrum analyser and is shown in Fig. 7. The right hand axis corresponds to the measured voltage (solid line). The difference in scale is due to the different drive amplitudes in the two cases. Again it is easy to identify the higher frequency modes, but the lower frequency modes are more difficult to spot. It is believed 


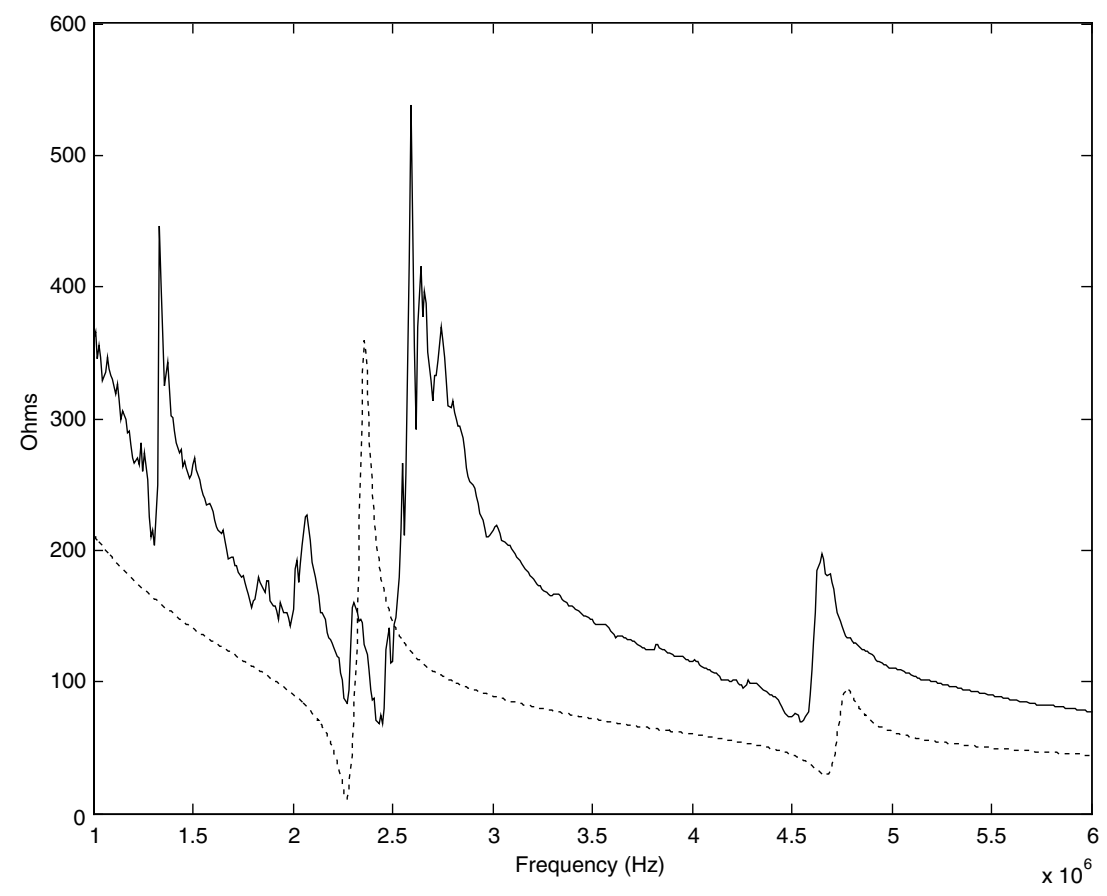

Fig. 5. Modelled (dotted) and measured (solid) impedance plots for the empty device.

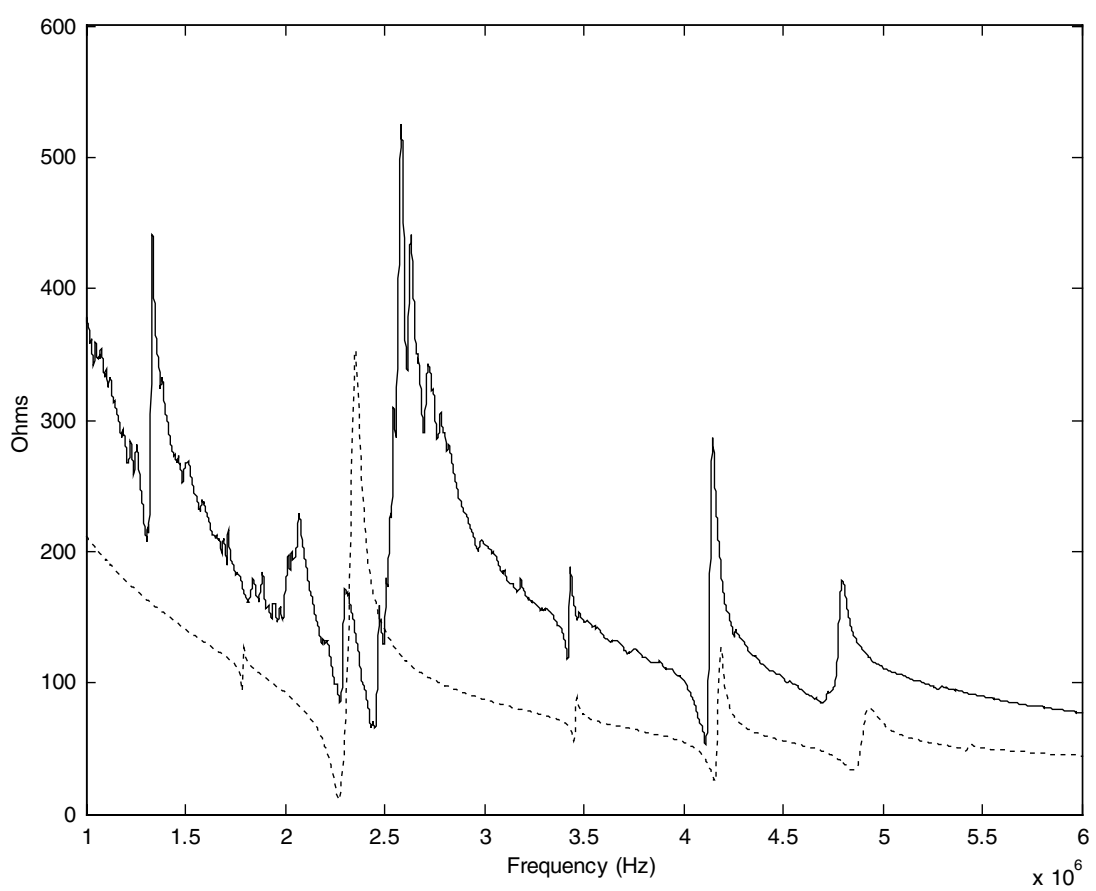

Fig. 6. Modelled (dotted) and measured (solid) impedance plots for the fluid filled device.

that the $2.2 \mathrm{MHz}$ mode is masked by the transducer resonance, but there is some evidence of the $1.8 \mathrm{MHz}$ mode, actually located at $1.714 \mathrm{MHz}$. Although disappointingly small, this mode proved to be experimentally locatable using an oscilloscope.

\section{Experimental results}

To verify the operation of the device, an optical microscope was used to measure the position of the particles within the chamber. The principle is that it is 


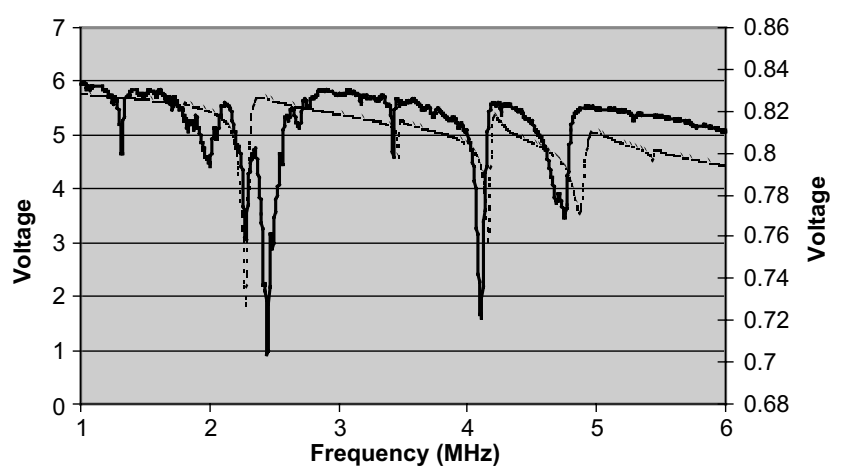

Fig. 7. Modelled (dotted) and measured (solid) voltage plots for the fluid filled device.

possible to focus on particles within a plane if the depth of focus is suitably small. By focussing on the silicon surface, and then the water/Pyrex boundary it is possible to generate a frame of reference.

The device was driven from a Frequency Synthesiser, via a fixed gain RF amplifier (50 dB). The transducer was placed in series with a small resistor $(47 \Omega)$ so that the combination acted like a potential divider. This was to allow the variation in voltage across the transducer to be monitored by an oscilloscope to allow voltage nulls to be identified. The frequencies of the expected modes were concentrated on. The chamber was gravity fed with a solution of yeast, and both still pictures and video clips were captured.

The technique of focussing the microscope proved successful, and all four modes could be identified. Modes were identified at 1.71, 2.27, 3.44 and $4.1 \mathrm{MHz}$, although as expected, mode 1 at $1.712 \mathrm{MHz}$ required extra power to identify. The sequence of images shown in Fig. 8 shows randomly distributed particles being forced to the Pyrex/water boundary against gravity as predicted for mode 1 at $1.712 \mathrm{MHz}$. Image (a) shows the random distribution of yeast before any power is applied, with the microscope focussed at the Pyrex/water boundary. Image (b) shows the view $5 \mathrm{~s}$ after the power is applied, and it can be seen that yeast cells are beginning to move into focus as they are forced against the boundary. Images (c) and (d) show the situations 5 and $10 \mathrm{~s}$ on, and many more cells are now in focus. By image
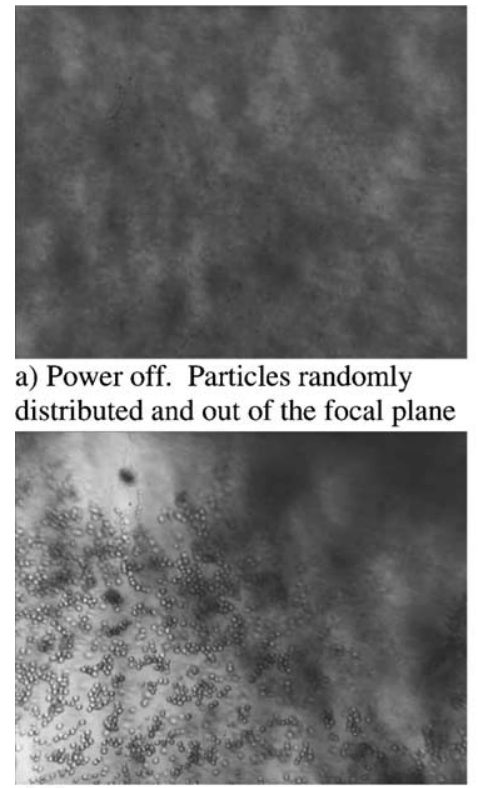

c) Power on.

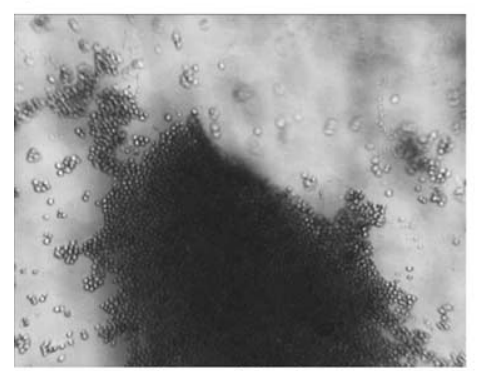

e) Power off. Particles begin to peel from reflector surface.

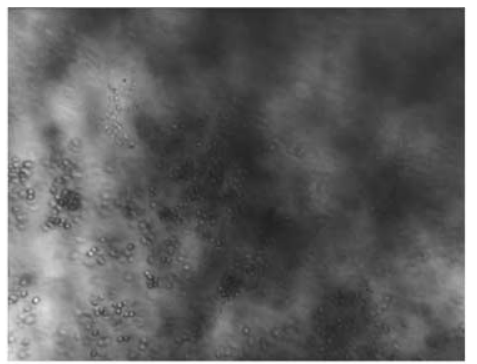

b) Power on. $60 \mathrm{mV}$ 1.7134MHz. Focus on reflector layer.

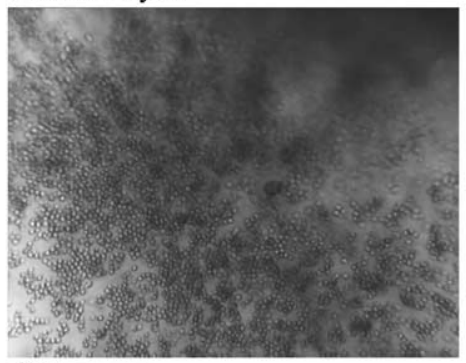

d) Power on.

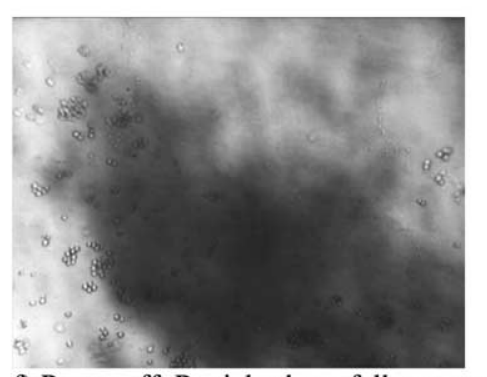

f) Power off. Particles have fallen away

Fig. 8. A sequence of images showing particle movement. 
(d) most particles are at the boundary. Particles tend to collect in clumps due to lateral forces, and image (e), taken $20 \mathrm{~s}$ later, just after the power has been turned off shows this. Image 6, taken 20 s later, shows the clump of particles peeling away from the Pyrex layer and falling under the influence of gravity towards the silicon layer and thus out of focus.

Similar results were obtained for the other three modes, showing that particles could be forced to either of the cavity boundaries or to the cavity centre.

\section{Conclusions}

In conclusion, an exercise which aimed to demonstrate a dual mode microfluidic ultrasonic particle manipulator has resulted in the design, fabrication and testing of a device which works at four modes within the frequency range of interest. Although the impedance and voltage responses of the quarter wave modes were less pronounced than expected, it still proved possible to identify these responses experimentally and indeed use them to manipulate yeast particles in the desired manner. The half wave modes were more obvious and also allowed particles to be concentrated in groups within a nodal plane in the centre of the cavity, rather than at a boundary, as in the case of the quarter wave modes.
This work has given very encouraging results and in the future, the model will be used to fine tune the parameters to allow better defined devices to be made.

\section{Acknowledgements}

This work has been supported by EPSRC grant number GR/R13333/01, DSTL, and Porvair PLC.

\section{References}

[1] M. Gröschl, Ultrasonic separation of suspended particles-Part I: fundamentals, Acustica 84 (1998) 432-447.

[2] M. Hill, R.J.K. Wood, Modelling in the design of a flow-through ultrasonic separator, Ultrasonics 38 (2000) 662-665.

[3] M. Hill, Y. Shen, J.J. Hawkes, Modelling of layered resonators for ultrasonic separation, Ultrasonics 40 (2002) 385-392.

[4] J.J. Hawkes, M. Gröschl, E. Benes, H. Nowotny, W.T. Coakley, Positioning particles within liquids using ultrasound force fields, in: 3rd EAA European Congress on Acoustics, Sevilla, 2002.

[5] M. Hill, The selection of layer thicknesses to control acoustic radiation force profiles in layered resonators, J. Acoust. Soc. Am. 114 (5) (2003) 2654-2661.

[6] N.R. Harris, M. Hill, S.P. Beeby, Y. Shen, N.M. White, J.J. Hawkes, W.T. Coakley, A silicon microfluidic ultrasonic separator, Sensors and Actuators B 95 (2003) 425-434. 